\title{
Frequency of sexually transmitted diseases and main methodological implications
}

\author{
Zaleida Napoli, Patrizia Lencioni, Margherita Niccolai, Riccardo Lari, Loria Bianchi \\ U.O. Laboratorio Analisi, Sezione Microbiologia, Ospedale "del Ceppo", Pistoia
}

Key words: Sexually transmitted diseases; HPV; NAATs; Real Time PCR; C. trachomatis; M. hominis, M. genitalium, U. parvum, U. urealyticum

\section{Frequenza delle principali malattie sessualmente trasmesse ed implicazioni metodologiche}

\section{SUMMARY}

Background. High risk Human Papillomavirus (HR-HPV) persistence is the most important cervical cancer risk factor, while Chlamydia trachomatis (CT), Neisseria gonorrhoeae (NG), Mycoplasma hominis (MH), Mycoplasma genitalium(MG), Ureaplasma urealyticum (UU) and parvum (UP) are sexually transmitted diseases (STDs) causing infertility, pregnancy complication, lung problems in newborns.

Methods. I 35 urine, I 35 urethral swabs, 553 cervical swabs, I I 0 seminal fluids and I 440 Thin Prep, were tested with culture methods, Real-Time PCR (RT-PCR) and multiplex SYBR Green PCR-endpoint to detect STDs. PCRendpoint was performed to detect HPV.

Results. Culture methods showed the lowest sensitivity: for MH it was only $24 \%$ (compared to RT-PCR). UP/UU were the most frequent pathogens (13\% with culture, $29 \%$ with PCR-endpoint, $41,67 \%$ with RT-PCR). Turn Around Time was respectively: $48 \mathrm{~h}, 6 \mathrm{~h}$ and $2 \mathrm{~h}$. RT-PCR cervical frequencies for CT, MH, MG, UU, UP were: $5.42 \%$, $11.03 \%, 1.81 \%, 11.21 \%$ and $35.08 \%$. HPV positivity in primary and secondary screening was $17.33 \%$ and $51.14 \%$. Highes $t$ positivity age group was: 23-32 years for CT (17\%), and $18-27$ years for HPV (33\%).

Conclusions. RT-PCR is more sensitive, faster, less expensive than other molecular tests like PCR-endpoint and microarrays. It allows more efficient laboratory organization: pre-analytical phase is more automated and enable the implementation of further diagnostic tests for pathologies that need rapid identification, such as meningitidis and sepsis, with reduced human and instrumental resource. Regarding STDs screening, it should be performed in women: for CT at least up to 27 years; for HPV between 35-50 years, since persisting HR-HPV infection is responsible of high-grade lesions.

\section{INTRODUZIONE}

Le infezioni sessualmente trasmesse (MST) hanno un'incidenza di circa 340 milioni di nuovi casi ogni anno (48); se non trattate, molte di esse possono produrre infezioni con gravi complicanze, quali infertilità, aborti precoci e malattie neonatali di grado severo $(18,24)$.

I costi socio-economici di queste infezioni e delle loro complicanze sono elevati; lo screening per la prevenzione primaria del cancro della cervice e gli interventi per il trattamento e follow-up delle neoplasie cervicali intraepiteliali di grado lieve (CIN 1), moderato (CIN 2) e grave (CIN 3), il trattamento dell'infertilità, la cura della cecità e delle infezioni polmonari nei neonati, rappresentano una larga porzione dei costi sanitari complessivi. Quantificare l'incidenza e la distribuzione di questi patogeni nella popolazione, mediante l'investimento in opportune strategie di controllo e prevenzione, è importante per ridurre la morbilità e la mortalità correlata a questo tipo di infezioni con ricadute, sicuramente, in termini di qualità di vita per i pazienti ma anche in termini di spesa sanitaria più complessa da calcolare. Numerosi sono $i$ test di amplificazione degli acidi nucleici (NAATs) in Real-Time polymerase chain reaction (RT-PCR) per la rivelazione di Chlamydia trachomatis e Neisseria gonorrhoeae (11, 23, 32, 45) mentre poco implementati quelli per la rilevazione di Mycoplasma genitalium, Mycoplasma hominis, Ureaplasma urealyticum e Ureaplasma parvum, nonostante numerosi studi abbiano dimostrato una maggiore sensibilità delle metodiche RT-PCR $(1,2,28,41,44)$. Diversi sono i laboratori che stanno implementando la diagnostica molecolare e impiegano le metodiche colturali solo quando è necessario l'antibiogramma. Praticamente inesistenti i test di diagnostica quantitativa in RT-PCR che hanno un'importanza nel follow-up del trattamento. Per la diagnostica del Papillomavirus umano ad alto rischio oncogeno (HR-HPV), numerose sono invece le metodiche NAATs per la rilevazione e la genotipizzazione basate sull'amplificazione del DNA virale delle

\section{Corresponding author: Loria Bianchi}

U.O. Laboratorio Analisi, Sezione Microbiologia, Ospedale “del Ceppo”, Pistoia

Viale Matteotti 9 - 5I 100 Pistoia - Tel: 0573-352055 - Fax: 0573-352474

E-mail: I.bianchi@usl3.toscana.it 
regioni virali L1 ed E1 (35). Pochi i test di diagnostica basati sull'amplificazione della regione codificante per le oncoproteine E6 ed E7, in grado di rilevare anche il DNA virale integrato, forma altamente frequente nei carcinomi e nelle lesioni intraepiteliali di alto grado (33). Pochi i lavori in letteratura che misurano la cellularità del campione, parametro importante per la valutazione dell' $i$ doneità del prelievo nella diagnostica molecolare soprattutto per la rilevazione di patogeni intracellulari quali HPV e C. trachomatis $(7,27,47)$.

Tale parametro, presente almeno qualitativamente nella lettura del Pap-test, non viene più valutato in un test molecolare quando eseguito su un prelievo dedicato. Tutti i test HPV hanno primers per l'amplificazione di una regione target di DNA umano che permette di valutare la presenza di cellule nel campione, ma non in grado di quantificarne la cellularità.

Obiettivi del seguente lavoro sono stati:

1) la valutazione di sensibilità e specificità delle metodiche NAATs rispetto alle metodiche "tradizionali” per patogeni quali Ureaplasma spp, Mycoplasma spp, N. gonorrhoeae e C. trachomatis;

2) la valutazione in termini di ricaduta clinica ed organizzativa delle metodologie scelte. A tale scopo i parametri valutati sono stati:

a) la frequenza delle coinfezioni;

b) la cellularità del campione per la valutazione dell'idoneità del prelievo a monte del processo;

3) stima della prevalenza delle principali MST, anche per fasce di età, per fornire ulteriori dati utili al disegno di strategie di screening più efficaci ed efficienti, soprattutto per C. trachomatis ed HR-HPV;

4) la valutazione della differenza di positività all'HPV nello screening primario e secondario e le probabili implicazioni metodologiche: due test, uno per lo screening I ed uno per lo screening II, o un solo test per entrambi?

\section{MATERIALI E METODI}

Popolazioni di studio. Nel nostro lavoro sono state analizzate tre popolazioni di soggetti:

1) Studio Pilota MST 1, una popolazione di 300 donne, di età compresa tra i 18 e i 58 anni, afferenti al laboratorio di Microbiologia nel periodo gennaio 2008 - gennaio 2009, per l'analisi di infezione genitale da $C$. trachomatis, $N$. gonorrhoeae, M. hominis e Ureaplasma spp su tampone cervicale con terreno di trasporto ESwab (Copan, Italy). Lo studio ha permesso di confrontare sensibilità e specificità delle metodiche NAATs rispetto alle metodiche "tradizionali" e l'analisi della prevalenza del virus HPV in una popolazione di screening primario;

2) Studio MST2, una popolazione di 933 individui, 306 uomini e 627 donne, afferenti al laboratorio di Microbiologia nel periodo gennaio 2010 - gennaio 2011, per l'analisi di infezione uro-genitale da C. trachomatis, $N$. gonorrhoeae, M. hominis, M. genitalium e Ureaplasma spp. I campioni consistono in: 135 urine, 110 liquidi seminali, 135 tamponi uretrali e 553 tamponi cervicali, da cui è stato estratto il DNA per la rilevazione dei patogeni. Lo studio ha permesso la stima della prevalenza delle principali MST nelle varie tipologie di prelievo e nelle varie classi di età;

3) Studio HPV, una popolazione di 1140 donne, di età compresa tra i 18 e i 70 anni, afferenti all'U.O. Anatomia Patologica, che presentavano al Pap-test lesioni intraepiteliali squamose di basso grado (L-SIL), di alto grado (H-SIL) e di incerto significato (ASCUS). Lo studio ha permesso l'analisi delle frequenze dei genotipi di HPV in una popolazione di screening di secondo grado, anche per fasce di età, ed il loro confronto rispetto ad una popolazione di screening primario (Studio MST1).

Estrazione del DNA. Il DNA presente nei campioni è stato estratto come da protocollo riportato nel kit EZ1 RNA/DNA Virus (Qiagen, Netherland). Il DNA estratto è stato eluito in un volume di $60 \mu \mathrm{l}$ e conservato a $-20^{\circ} \mathrm{C}$ fino all'analisi.

Metodiche di riferimento. I 300 campioni dello studio MST1 sono stati sottoposti ad indagine mediante le metodiche utilizzate di routine nel laboratorio di Microbiologia. In particolare, per M. hominis e Ureaplasma spp è stato utilizzato il terreno di coltura agar A7 e il test Mycoplasma IST 2 (bioMérieux, France), per $N$. gonorrhoeae il terreno Thayer Martin VCA (bioMérieux, France), per C. trachomatis il test molecolare BD ProbeTec (BD, USA), basato sulla tecnica strand displacement amplification (SDA).

PCR endpoint "home made". Il DNA estratto dai 300 tamponi cervicali E-Swab dello studio MST1 è stato sottoposto ad analisi mediante una PCR endpoint "home-made" per la rilevazione di C. trachomatis, N. gonorrhoeae, M. hominis, Ureaplasma spp. I primers specifici usati per amplificare il genoma dei quattro patogeni e per il controllo di amplificazione sono riportati in Tabella 1 (Eppendorf, Germany). Le reazioni di amplificazione sono state eseguite mediante utilizzo di Sybr Greneer Universal master mix (Life Technologies). I patogeni sono stati amplificati su termociclatore Rotorgene Q (Qiagen) con il seguente profilo termico: 1 ciclo a $95^{\circ} \mathrm{C}$ per 10 minuti, seguito da 40 cicli di denaturazione a 
$95^{\circ} \mathrm{C}$ per 15 secondi, annealing a $54^{\circ} \mathrm{C}$ per 15 secondi ed estensione a $72^{\circ} \mathrm{C}$ per 30 secondi; il target del patogeno amplificato è stato identificato mediante analisi della curve di melt eseguite a fine amplificazione. La messa a punto della metodica è stata effettuata utilizzando ceppi di riferimento di C. trachomatis (ATCC VR878), M. hominis (ATCC 14268), N. gonorrhoeae (ATCC 19424), Ureaplasma parvum (ATCC 27815) e Ureaplasma urealyticum (ATCC 29559).

PCR Real-Time. Il DNA estratto dai 933 campioni dello studio MST2 è stato sottoposto all'analisi in RT-PCR per M. hominis, M. genitalium, $U$. urealyticum, $U$. parvum, C. trachomatis e $N$. gonorrhoeae. Per M. hominis (regione target: rRNA 16s; MYCOPLASMA HOMINIS RealTime, Nuclear Laser) e M. genitalium (regione target: gyrB; MYCOPLASMA Genitalium RealTime, Nuclear Laser) la metodica è a singolo patogeno, per $U$. urealyticum e $U$. parvum (regione target: ureaC) la metodica è in multiplex (UREAPLASMA Parvum/Urealyticum Real-Time, Nuclear Laser). Tutti i patogeni sono stati amplificati con un unico profilo termico che è il seguente: 15 minuti a $95^{\circ} \mathrm{C}$, seguito da: primi 10 cicli composti da $95^{\circ} \mathrm{C}$ per 20 secondi, $65^{\circ} \mathrm{C}$ per 20 secondi e $72^{\circ} \mathrm{C}$ per 20 secondi; successivi 35 cicli composti da $95^{\circ} \mathrm{C}$ per 20 secondi, $60^{\circ} \mathrm{C}$ per 30 secondi e $72^{\circ} \mathrm{C}$ per 15 secondi. Per C. trachomatis la metodica RT-PCR usata è a singolo patogeno e le regioni target amplificate sono due: il gene che codifica la proteina maggiore di membrana (MOMP) e una regione del plasmide criptico (C. trachomatis Plus RG PCR, Qiagen, Germany). Per N. gonorrhoeae la metodica usata (Anyplex CT/NG RT, Seegene, Korea) è a doppio patogeno e le due regioni target amplificate sono relative ai geni porA (porine di membrana di classe I) e mngo (metilasi NgoPII). I risultati positivi sono stati riconfermati con un altro test a 2 regioni target, opa e porA (Expeteam, Italia).

L'intero processo di analisi è validato mediante controllo interno presente in tutti i kit. Il laboratorio aderisce al controllo esterno di qualità NEQAS per C. trachomatis, $N$. gonorrhoeae e HPV.

Idoneità del campione. L'idoneità dei campioni dello studio MST2 e di 332 campioni dello studio HPV è stata valutata mediante amplificazione in RT-PCR di una porzione del gene umano codificante per l'enzima ipoxantina-guanina fosforibosiltransferasi 1 (HPRT1; Cell Control r-gene, Argene, France) e quantificazione mediante utilizzo di 3 standard a concentrazione nota. Il profilo termico di amplificazione è il seguente: 15 minuti a $95^{\circ} \mathrm{C}$, seguito da 45 cicli composti da 10 secondi a $95^{\circ} \mathrm{C}, 30$ secondi a $60^{\circ} \mathrm{C}$ e 10 secondi a $72^{\circ} \mathrm{C}$. Per interpolazione sulla retta standard, è stata calcolata la quantità di cellule iniziali presenti nel campione. La valutazione di questi dati ha permesso: a) di comparare la cellularità presente nei tamponi cervicali E-Swab con quelli ThinPrep; b) indirettamente di comparare la cellularità così calcolata con quella valutata con la lettura del Pap-test.

Ricerca di HPV e genotipizzazione. Il DNA estratto dai tamponi cervicali relativi agli studi HPV e MST1 è stato amplificato per la ricerca di HPV mediante test INNO-LiPA HPV Genotyping Extra (Innogenetics, Belgium). La metodica usata è una classica PCR endpoint e il set di primers biotinilati SPF10 hanno come target la regione target L1 (65 pb). Contemporaneamente viene amplificato un frammento del gene umano HLADBP1 che serve come controllo qualitativo di cellularità (controllo di inibizione/cellularità).

La genotipizzazione dei ceppi di HPV (genotipi rilevati: 27) è stata effettuata con metodica di ibridazione inversa su striscia e i passaggi di ibridazione degli amplificati con le sonde sono stati completamente automatizzati mediante l'utilizzo dello strumento Auto-Lipa (Innogenetics, Belgium).

\section{RISULTATI}

Studio pilota MST 1 - Valutazione delle frequenze delle principali malattie sessualmente trasmesse. In tabella 2 sono stati riportati i casi positivi rilevati (A) e la frequenza riscontrata (B) per ciascun patogeno nei 300 tamponi cervicali analizzati mediante le varie tecniche. La frequenza di positività per ogni singolo patogeno varia a seconda della metodica impiegata. La specie Ureaplasma è quella più rappresentata (dal 13\% al $41.67 \%$ ); $M$. hominis è stato rilevato nel 2.67 $11.33 \%$ dei casi analizzati, C. trachomatis nel 4$5.67 \%$, N. gonorrhoeae nell' $1.33-7.33 \%$.

Considerando come metodica gold standard per ogni patogeno analizzato la RT-PCR, la metodica colturale è risultata la meno sensibile fra tutte le metodiche impiegate per tutti i patogeni indagati, con una sensibilità che varia dal $24 \%$ per $M$. hominis al 33\% per N. gonorrhoeae (tabella 3).

Per N. gonorrhoeae la PCR multiplex home-made ha mostrato una specificità ridotta rispetto alla metodica colturale e alla Real-Time PCR (96\%).

Studio MST 2 - Confronto delle frequenza nelle diverse matrici biologiche. Le frequenze riscontrate mediante utilizzo della metodica RealTime PCR nei 933 campioni analizzati sono mostrate nella tabella 4. Nei soggetti di sesso maschile i patogeni più frequenti sono rappresentati da C. trachomatis (rilevata nel $15.69 \%$ dei casi) e U. parvum (rilevato nel $14.38 \%$ dei casi). La frequenza più elevata per C. trachomatis viene riscontrata nei tamponi uretrali (23.58\%), mentre 
quella più bassa nelle urine $(5.56 \%)$.

La frequenza più elevata per $U$. parvum viene riscontrata nel liquido seminale (18.18\%), mentre la frequenza riscontrata nei tamponi uretrali è similare a quella riscontrata nelle urine ( $12.26 \%$ e $12.22 \%$ rispettivamente).

$U$. urealyticum, $M$. hominis e $M$. genitalium sono rilevati rispettivamente nel $6.21 \%, 5.56 \%$ e $3.27 \%$ dei casi.

Nei soggetti di sesso femminile $U$. parvum, rilevato nel $34.29 \%$ dei casi totali, è il patogeno ritrovato più frequentemente in tutte le matrici analizzate. L'infezione da $C$. trachomatis è presente nel $5.1 \%$ dei casi, con una positività massima rilevabile nei tamponi cervicali $(5.42 \%)$ e minima nelle urine $(2.22 \%)$. U. urealyticum, M. hominis e $M$. genitalium presentano una frequenza globale del $10.53 \%, 10.37 \%$ e $1.75 \%$ rispettivamente.

Studio MST 2 - Valutazione delle coinfezioni. Nei 933 campioni analizzati mediante impiego di test in RT-PCR è stata riscontrata una correlazione statisticamente significativa tra le infezioni causate da M. hominis, U. urealyticum e $U$. parvum $\left(p<0.005\right.$ con test del $\chi^{2}-$ tabella 5).

Il $43.53 \%$ dei campioni positivi a $U$. urealyticum presentano anche un'infezione da U. parvum ed il $18 \%$ da $M$. hominis, contro una percentuale generale di infezione del $27.76 \%$ e $8.79 \%$ rispettivamente. Il $41.46 \%$ dei campioni positivi a $M$. hominis presenta anche un'infezione da U. parvum; il $19.51 \%$ presenta invece una coinfezione con U. urealyticum, mostrando una frequenza doppia rispetto alla percentuale globale del $9.11 \%$. L'analisi dei dati rivela anche una correlazione statisticamente significativa tra i due patogeni C. trachomatis e M. genitalium $(p<0.025$; tabella 5): il $23.81 \%$ dei campioni positivi a $M$. genitalium presentano una contemporanea infezione di C. trachomatis, contro una percentuale globale di infezione da C. trachomatis dell' $8.57 \%$. Studio MST 2 e Studio HPV - Valutazione delle frequenze di infezione in relazione all'età.

I soggetti analizzati hanno un'età compresa fra $\mathrm{i}$ 18 e i 58 anni e le classi di età sono quelle riportate nelle tabelle 7 e 8 , a seconda del patogeno considerato. Tra i soggetti di sesso maschile non è stata riscontrata nessuna correlazione statisticamente significativa con l'età per nessuno dei patogeni indagati (dati non mostrati). Tra i soggetti di sesso femminile è stata invece riscontrata un'associazione statisticamente significativa $(p<0.005)$ tra infezione da $C$. trachomatis ed età: il $10.97 \%$ dei soggetti di età compresa tra i 23 ed i 32 anni risultano positivi al patogeno contro un tasso globale del $5.10 \%$. Altra associazione statisticamente significativa è stata riscontrata per l'infezione da $U$. urealyticum $(p<0.05)$ : i soggetti con età infe- riore ai 23 anni (con un tasso di infezione del $21.57 \%$ ) mostrano una frequenza significativamente aumentata rispetto al tasso globale del $10.53 \%$. Le infezioni da $U$. parvum, M. hominis e $M$. genitalium non mostrano una significativa variazione delle frequenze rispetto al tasso globale in nessuna classe di età analizzata.

I risultati ottenuti dall'analisi per la ricerca di HPV condotta su 300 campioni provenienti dallo screening primario e 1140 campioni provenienti dallo screening secondario sono illustrati in tabella 6. Le due popolazioni di studio presentano una differenza statisticamente significativa nella frequenza dell'infezione da HPV (17.33\% nello screening primario contro il $51.14 \%$ dello screening secondario, $p<0.005$ ).

Nella popolazione di screening primario la distribuzione della frequenza dell'infezione da HPV mostra una significativa variazione in relazione all'età dei soggetti sottoposti ad analisi $(p<0.05$, tabella 8 ). In particolare i soggetti di età compresa tra i 18 ed i 22 anni mostrano una frequenza di infezione pari al $33.3 \%$ e quelli di età compresa tra i 23 ed 28 anni pari al $36.7 \%$, con una prevalenza dell'infezione doppia rispetto alla frequenza globale che è del $17.33 \%$. Anche la popolazione di screening secondario mostra una significativa variazione della frequenza in relazione all'età $(p<0.025)$; tuttavia i soggetti nei quali è riscontrata la maggiore frequenza dell' infezione $(60 \%$ contro una frequenza globale del $51.14 \%$ ) sono quelli di età compresa tra i 28 ed i 32 anni.

Valutazione dell'idoneità del prelievo. Nella tabella 9 è riportata la cellularità media/prelievo $(\mathrm{Cm} / \mathrm{p})$, la deviazione standard, l'errore standard e l'intervallo di confidenza al $95 \%$ per le tipologie di prelievo e matrice biologiche analizzate. È stata confrontata la $\mathrm{Cm} / \mathrm{p}$ fra il prelievo cervicale nel contenitore ThinPrep e quello nella provetta E-Swab. La prima tipologia di contenitore è usata nei laboratori di Anatomia Patologica e permette l'esecuzione del Pap-test con la citologia su strato sottile, con la possibilità di eseguire esami di approfondimento come quelli della ricerca di eventuali patogeni (HPV, C. trachomatis, ecc) in biologia molecolare. Le provette ESwab, similmente, sono molto usate nei laboratori di batteriologia/virologia in quanto permettono di eseguire esami batteriologici colturali con seminatore automatico e, con lo stesso prelievo, esami di biologia molecolare. Il cut-off proposto in tabella 9 equivale a circa il 10\% della numerosità cellulare media ottenuto per quel tipo di matrice biologica. I tamponi cervicali hanno la $\mathrm{Cm} / \mathrm{p}$ massima mentre i campioni a densità cellulare più bassa sono quelli di tipo urinario. Utilizzando il cut-off proposto, la percentuale dei campioni non idonei è stata del 
$19.33 \%, 13.47 \%, 14.81 \%$ e $26.67 \%$ per i tamponi cervicali, tamponi uretrali ed urine rispettivamente.

\section{DISCUSSIONE}

Anche per la diagnostica microbiologica, come per altri settori quali quello della diagnostica oncologica, la biologia molecolare si sta affermando sempre di più. Le varie tecniche di biologia molecolare consentono, con una maggiore sensibilità, di rilevare la presenza di agenti patogeni virali, batterici e micotici in campioni biologici, in alcuni casi sostituendo le metodiche tradizionali, in altri aggiungendosi alle stesse, in altri ancora rappresentando le uniche metodiche disponibili $(26,29)$. Negli ultimi anni il settore molecolare applicato alla diagnostica microbiologica è stato fra quelli che ha avuto maggiore sviluppo, sia come entità di test immessi sul mercato, più $o$ meno appropriati, sia come evoluzione tecnologica, soprattutto con lo sviluppo di microarray per patologie gravi quali sepsi, polmoniti e meningiti (4), dove esiste la necessità di testare il più rapidamente possibile tutta una serie di patogeni responsabili di tali patologie per instaurare tempestivamente una terapia mirata con migliore prognosi per il paziente. Nelle strategie aziendali di razionalizzazione della spesa, sempre più si presta attenzione all'appropriatezza della richiesta e alla corretta gestione clinica del paziente secondo percorsi diagnostici condivisi per aumentare l'efficacia del percorso terapeutico intrapreso. Ad esempio, esiste numerosa letteratura scientifica che mette in evidenza come il test HPV, utilizzato nella gestione degli ASCUS e degli L-SIL, nel follow-up delle pazienti conizzate e come test aggiuntivo al Pap-test in donne con età superiore ai 30 anni, permetta un ritorno economico in termini di riduzione del numero di colposcopie ed incremento di lesioni intraepiteliali rilevate in fase precoce (30).

Per la razionalizzazione della spesa sempre più attenzione deve essere prestata anche all'introduzione dei nuovi test di biologia molecolare che possono portare ad un rapporto costo/beneficio inappropriato se non vengono valutati attentamente in termini di:

a) qualità analitica e ricaduta clinica per il paziente;

b) organizzazione dell'attività diagnostica (service dedicati per singolo patogeno piuttosto che piattaforme integrate per rilevazione di più patogeni con PCR multiplex e/o simultanee);

c) appropriatezza del test (ad esempio scelta di PCR multiplex con pannelli di patogeni adeguati al quesito diagnostico).

Numerose sono le metodiche NAATs considerate il gold standard, validate ed automatizzate su piattaforma dedicata, per la ricerca di C. trachomatis $(11,23)$. L'elevata sensibilità $(>90 \%)$ e specificità $(>98 \%)$ rendono questi metodi i più appropriati per la ricerca di questo patogeno.

L'uso di metodi non-NAATs, soprattutto nello screening di popolazioni a medio-basso rischio o asintomatiche, si traduce in una diminuzione nella stima della prevalenza dell'infezione fino al 30$40 \%$ (6).

Per la rilevazione di micoplasmi e ureaplasmi pochi sono i metodi PCR endpoint e rari quelli in RT-PCR $(15,25)$ in quanto M. hominis, U. urealyticum e U. parvum, batteri commensali del tratto genitale femminile, sono stati per lungo tempo considerati a bassa virulenza. Tuttavia, poiché numerosi studi hanno dimostrato una significativa associazione fra questi microrganismi e complicanze anche gravi quali infertilità femminile (20) e maschile (50), aborti precoci $(22,24)$, nascite premature (49) e malattie neonatali quali infezioni del tratto respiratorio superiore, polmoniti e meningiti (46), anche per questi patogeni le metodiche NAATs hanno avuto negli ultimi anni una notevole implementazione. Se per C. trachomatis soltanto i test NAATs sono raccomandati dalle linee guida europee (23), per quanto riguarda M. hominis e Ureaplasma spp il gold standard è ancora la metodica colturale. Tale metodica permette di eseguire anche l'antibiogramma con i limiti, però, di avere una minore sensibilità (tabelle 2 e 3) e di non rilevare $M$. genitalium, che deve essere ricercato comunque con metodiche NAATs in quanto non coltivabile (38). Con le tecniche "tradizionali" i tamponi necessari per uno screening cervicale completo per MST possono essere fino a 4 :

a) uno per C. trachomatis rilevata con metodica dedicata SDA;

b) uno per la ricerca di micoplasmi/ureoplasmi e relativo antibiogramma con sistema colturale IST 2 o semina su piastre di agar A7;

c) uno per M. genitalium, HPV, HSV $1 / 2$ rilevati con test in RT-PCR; d) uno per N. gonorrhoeae da ricercare con metodica colturale. È stato dimostrato che l'impiego di più tamponi eseguiti in successione determina un aumento del numero di falsi negativi (10-20\%), dato che correla in maniera statisticamente significativa $(p<0.01)$ con la cellularità del campione (dati non riportati).

Come infatti si può osservare dalla tabella 3 , ottenuta con i dati dello studio pilota MST 1, emerge che: a) la metodica meno sensibile per tutti i patogeni analizzati (Ureaplasma spp, M. hominis e $N$. gonorrhoeae) è la metodica colturale. La bassa sensibilità del metodo colturale è da attribuirsi anche alla labilità dei germi studiati (cosiddetti 
germi "esigenti") che non riescono a sopravvivere a lungo nel mezzo di trasporto. Tale dato è supportato anche dai dati ottenuti con il sistema IST 2 dove la sensibilità del metodo è del $58 \%$ vs $31 \%$ per Ureaplasma spp e $44 \%$ vs $24 \%$ per M. hominis. Per tutti i patogeni analizzati la metodica più sensibile è risultata la RT-PCR; b) per $N$. gonorrhoeae la positività maggiore rilevata con la $\mathrm{PCR}$ endpoint rispetto alla RT-PCR (7.33\% vs $4 \%)$ è dovuta alla presenza di falsi positivi per cross-reattività dei primers scelti con altre specie di Neisserie commensali e/o non patogene, come dimostrato dalle riconferme $(4 \%)$ con le metodiche RT- PCR (Diagenode, Belgium; Anyplex, Seegene). Si sottolinea, quindi, l'importanza, almeno per questo patogeno, di riconfermare i positivi con un test che impiega un target diverso da quello del test di screening e di impiegare, quando disponibili, metodiche con primers target per due diverse regioni del genoma per incrementare la sensibilità (8). Altro limite dei metodi molecolari per questo patogeno è quello di non permettere la sorveglianza della resistenza agli antibiotici come, ad esempio, la ridotta sensibilità ai chinoloni riportata per i ceppi importati dall'Asia. Lo studio pilota MST 1 ha inoltre permesso di rilevare l'alta prevalenza di infezioni causate da M. hominis e U. urealyticum e la bassa prevalenza di infezione per patogeni quali $C$. trachomatis e $M$. genitalium, tutti patogeni per i quali è importante contenere la trasmissione per le patologie causate precedentemente descritte. Per tale motivo emerge che l'introduzione di metodiche PCR multiplex per patogeni che hanno sito di infezione comune potrebbe portare ad un miglioramento della diagnostica delle MST con un alto ritorno qualitativo in termini di salute. Quanto detto viene ulteriormente confermato dallo studio MST 2 condotto su un totale di 933 individui (627 donne e 106 uomini). Nonostante i tassi di prevalenza riscontrati nella popolazione siano molto variabili e dipendano dalla zona geografica, dalle tecniche utilizzate, dall'ampiezza del campione analizzato ed anche dalle modalità di estrazione del campione, le frequenze rilevate per C. trachomatis, M. hominis, M. genitalium, U. parvum (ex- U. urealyticum biovar 1) e $U$. urealyticum (ex- U. urealyticum biovar 2) sono rapportabili a quelle riportate in letteratura. Nel nostro studio i batteri più comunemente rilevati in tutte le popolazioni prese in esame sono gli Ureaplasma, con $U$. parvum che raggiunge il range di positività più alto, in accordo con i lavori presenti in letteratura $(21,34,36)$. Stessa concordanza con la letteratura (range di positività: $1.3 \%-21.9 \%$ ) si trova per la positività di $M$. hominis che è del $10.37 \%$ nei tamponi cervicali $(5,21,34,36)$; negli uomini (tampone uretrale e liquido seminale) M. hominis si ritrova rispettivamente nel $5.6 \%$ ed $8 \%$, con una positività leggermente superiore rispetto a quella riportata in letteratura $(39,43)$. Anche la positività a $M$. genitalium, che è del $1.75 \%$, è in accordo con quanto riportato in letteratura $(5,34,36,39,43)$. L'inserimento in routine dell'estrazione automatica del DNA con o senza pretrattamento del campione ha permesso ai "nuovi" test NAATs di essere impiegati anche in campioni alternativi al tampone cervicale o uretrale quali liquido spermatico e soprattutto urine, matrice biologica di elezione per lo screening soprattutto nell'uomo $(11,17)$. Numerosi sono infatti gli studi condotti sulla tipologia di matrici su cui ricercare i diversi patogeni e la maggior parte riguardano la ricerca di C. trachomatis sulle urine (16). Praticamente inesistenti sono però i lavori in letteratura che valutano il pretrattamento del campione, la modalità estrattiva e le modalità di prelievo misurando la cellularità del campione, mentre quasi tutte le metodiche valutano la presenza di inibitori nell'estratto mediante l'impiego di un controllo interno.

Dai dati riportati dallo studio MST 2 emerge che per C. trachomatis, U. urealyticum, U. parvum, $M$. hominis la positività più bassa, fra tutte le matrici osservate, si ha sempre nelle urine.

Ad esempio, nei maschi, si osserva una positività a C. trachomatis del $23.58 \%$ nel tampone uretrale, comparabile con la positività riscontrata nel liquido seminale $(17.3 \%)$, mentre la positività nelle urine è solo del 5.56\% (tabella 4). Questo dato non supporta i numerosi lavori in letteratura che riportano le urine come la matrice più indicata per effettuare gli screening nei maschi con una sensibilità dell' $85-95 \%(11,17)$. Inoltre, nello studio MST2, tutta la messa a punto della fase di prelievo ed estrazione è stata settata confrontando la cellularità/prelievo utilizzando la quantificazione di un gene umano mediante Real-Time PCR. Come è possibile osservare dalla tabella 9 , la $\mathrm{C} / \mathrm{p}$ presente nei tamponi cervicali utilizzando i contenitori ThinPrep (per citologia su strato sottile) è comparabile a quella che si ha utilizzando i tamponi E-Swab, utilizzati in microbiologia per la semina automatica e la ricerca di altri patogeni a sede cervicale. Si può inoltre osservare che la $\mathrm{C}_{\mathrm{m}}$ minore si osserva per le urine, inferiore di circa 5 volte a quella ritrovata nel tampone uretrale e cervicale. Per poter valutare l'idoneità del prelievo e stimare l'entità dei risultati falsi negativi dovuti alla scarsa cellularità del campione, si è inserito un cut-off quantificabile per tutte le matrici analizzate. Si osserva come in base ai cut-off scelti, per tampone cervicale ThinPrep, cervicale E-swab, uretrale ed urine i prelievi inadeguati sono del $19 \%, 13 \%, 14 \%$ e $26 \%$ rispettivamente.

Sempre dallo studio MST 2 emerge che: 1) esiste 
una correlazione statisticamente significativa (tabella 4) tra le infezioni causate da M. hominis, $U$. urealyticum e $U$. parvum $(p<0.005) ; 2)$ esiste una correlazione statisticamente significativa fra C. trachomatis e M. genitalium $(p<0.025)$ e nello studio MST 1 una correlazione statisticamente significativa esiste anche fra $C$. trachomatis e $N$. gonorrhoeae $(p<0.05)$. Ancora, dallo studio MST 2 risulta che nelle donne esiste una correlazione statisticamente significativa $(p<0.005)$ tra infezione da $C$. trachomatis e classi di età (tabella 7), mentre per gli uomini, pur osservando una maggiore infezione in tale fascia di età, non si raggiunge la significatività statistica probabilmente per il ridotto numero di campioni. La positività osservata è del $10.94 \%$ nei soggetti con età compresa tra i 23 e 32 anni contro un tasso globale di positività del $5.10 \%$. Ne consegue che lo screening per questo patogeno andrebbe effettuato almeno per le donne fino a 30 anni ed esteso anche agli uomini (stessa fascia di età).

Negli ultimi anni un ruolo di primo piano nelle MST lo svolge la diagnostica molecolare dell'HPV, che sta diventando il test di prima scelta per lo screening della cervice uterina $(9,13$, 37). I metodi NAATs per la ricerca di HPV sono numerosissimi, con regioni target e metodiche diverse (35). Il test più utilizzato è l'Hybrid Capture 2 (HC2) della Digene Corp., approvato dalla Food and Drug Administration (FDA) nel 2003 per la rilevazione dell'infezione da HPV ad alto rischio per il triage delle donne con citologia ASCUS e come test di screening di primo livello, da associarsi alla citologia tradizionale, per le donne di età superiore ai 30 anni (35). Questo test permette la rilevazione di 13 genotipi di HPV ad alto rischio e 5 a basso rischio attraverso l'ibridazione del campione con una miscela di sonde; non permette l'esatta determinazione del tipo di HPV presente nel campione, ma esprime solamente la presenza o assenza degli HPV testati. Tuttavia, la grande varietà di test che continuano ad essere immessi sul mercato per la diagnostica dell'HPV, è sostanzialmente dovuta all'assenza di un test ideale per la rilevazione e genotipizzazione di questo virus responsabile del cancro della cervice uterina (3).

Le differenze principali tra $\mathrm{i}$ diversi test presenti sul mercato riguardano:

a) la metodica usata (PCR-endpoint, utilizzata da gran parte dei test di genotipizzazione con ibridazione su striscia; Real-Time PCR, come l'Abbott RealTime High Risk HPV assay; tecnologia microarray, come l'Infiniti HPV assay dell'AutoGenomics), il tipo di target (DNA/RNA) e regione virale bersaglio (L1, E1, E6/E7), la diversa abilità di discriminare tra infezioni singole e multiple, la capacità più o meno limitata di individuare solo un certo numero di genotipi e di rilevare anche il DNA integrato $(13,14,19$, 37, 42). La positività all'HPV (tabella 6) rilevata nel nostro studio nella popolazione di screening primario (17.3\%), mediante utilizzo del kit Innolipa Genotyping Extra, è risultata comparabile a quella riscontrata in numerosi studi effettuati in Europa e in Nord America, basati sull'utilizzo del test $\mathrm{HC} 2$ o di primers rivolti verso la regione L1 (13). Nella popolazione di screening secondario è stata invece osservata una positività nettamente superiore $(51 \%)$, d'altronde comparabile con quella riscontrata in studi effettuati su popolazioni di soggetti che presentavano alterazioni al Pap-test, effettuati sempre con il test HC2 (19). La differenza riscontrata nella positività ad HPV tra screening primario e secondario risulta statisticamente significativa $(p<0.005)$. Differente è anche la distribuzione della frequenza di positività, statisticamente significativa, per fasce di età (tabella $8)$. Nello screening primario $(p<0.05)$ la frequenza maggiore si ha nelle fasce di età 18-22 anni (33.3\%) e 23-28 anni (36.7\%) mentre nello screening secondario $(p<0.025)$ la frequenza maggiore si ha nella fascia di età $28-32$ anni $(60 \%)$. Da questo ne consegue che, essendo la persistenza dell'infezione virale la causa delle lesioni cervicali di alto grado (10), per incrementare la sensibilità del Pap-test nel rilevare le lesioni di alto grado, il virus andrebbe ricercato almeno nelle donne di età compresa fra i 35 e i 50 anni.

\section{CONCLUSIONI}

La RT-PCR è ad oggi considerata la metodica gold standard per la ricerca di C. trachomatis (23) e M. genitalium (38) e sta diventando tale anche per M. hominis, U. urealyticum e U. parvum.

Un test in RT-PCR presenta infatti:

a) sensibilità, specificità e riproducibilità più elevate rispetto ad un test in PCR endpoint;

b) non richiede manipolazioni post-amplificazione con conseguente diminuzione dei tempi e dei rischi di contaminazione ambientale;

c) rappresenta uno strumento prezioso per migliorare la diagnostica e fornire al clinico risultati attendibili e in tempi utili $(<2 \mathrm{~h})$;

d) può aiutare il clinico nel follow-up terapeutico quantificando la carica del patogeno.

Per $N$. gonorrhoeae, nella pratica clinica, i metodi molecolari non dovrebbero essere utilizzati come unico saggio diagnostico di routine, ma dovrebbero sempre essere confermati dall'esame colturale mediante richiamo delle donne positive alle NAATs $(8,48)$.

Come riportato in precedenza, per la diagnostica molecolare di $N$. gonorrhoeae bisogna utilizzare metodi molecolari con doppio target di DNA 
genomico in quanto si possono avere falsi positivi dovuti a cross-reazioni con Neisserie commensali come, ad esempio, quelle presenti nella cavità orale (elevata omologia di sequenze con $N$. meningitidis) e ad altre Neisserie non patogene (8). Altra limitazione dei metodi molecolari per questo patogeno è quella di non permettere di sorvegliare la resistenza agli antibiotici, come ad esempio la ridotta sensibilità ai chinoloni riportata per i ceppi importati dall'Asia. Vista la frequenza di positività a $M$. hominis, $U$. urealyticum e $U$. parvum, la correlazione che esiste fra $\mathrm{i}$ tre patogeni $(p<0.005)$ e quindi il numero di campioni positivi per almeno 2 di questi patogeni (tabella 5), l'impiego di una RT- PCR multiplex potrebbe essere la scelta metodologica ottimale per rilevarli con migliore razionalizzazione delle risorse economiche. Inoltre, vista la correlazione statisticamente significativa anche fra $C$. trachomatis e $M$. genitalium $(p<0.025)$ e $C$. trachomatis e $N$. gonorrhoeae $(p<0.05)$ e la bassa frequenza di positività per questi patogeni, anche qui l'impiego di una RTPCR multiplex potrebbe essere la scelta metodologica da seguire, con ulteriore riduzione dei tempi tecnici e dei costi lavorando su pool di campioni come già dimostrato $(12,40)$. La riduzione dei costi $(50-63 \%)$ e del tempo tecnico di esecuzione (50-60\%) risultano ottimali quando il pool è di 4-5 campioni. Da questa analisi statistica risulterebbe che l'impiego di RT-PCR multiplex a più di 2-3 patogeni porta ad una inappropriatezza della richiesta perché: a) ricercati come screening anche in assenza di sintomatologia clinica o fattori di rischio; b) ricercati anche in corso di followup nei pazienti positivi per un altro patogeno. Questo comporta un incremento di spesa che può arrivare fino al $30 \%$; c) diminuzione della qualità analitica in termini di sensibilità quale limite intrinseco della RT-PCR multiplex. Mantenendo le stesse modalità estrattive, il kit NG/CT Anyplex (Seegene, Korea), a doppio patogeno, ha dato un minor numero di campioni positivi (decremento del $3 \%$ ) non rilevando come positivi i campioni che hanno presentato un ciclo soglia (ciclo di amplificazione al quale si raggiunge l'inizio della fase esponenziale) $>39$ con il kit Artus (Qiagen) a singolo patogeno. La differenza in termini di sensibilità fra $i$ due test è stata confermata anche con i controlli NEQAS ed è stata quantificata in 45 cicli soglia. La stessa differenza in sensibilità è stata osservata anche fra i due kit utilizzati per $N$. gonorrhoeae e la maggiore sensibilità è stata osservata per la metodica in RT-PCR a singolo patogeno.

Le implicazioni metodologiche che derivano dalla diversa positività al test HPV osservata fra screening I e II (tabella 6) sono le seguenti:

per uno screening primario possono essere necessarie due differenti metodiche: a) un test come il test HC2 (Digene) che permetta di rilevare la presenza dei 13 genotipi ad alto rischio, senza genotipizzare; b) un test per la rilevazione e la genotipizzazione (con rilevazione delle coinfezioni) dei casi positivi al primo e che presentano alterazioni intraepiteliali ASCUS, L-SIL, H-SIL al Pap-test e CIN 1, CIN 2 e CIN 3 all'esame istologico. La bassa prevalenza dell'HPV nello screening primario non permette l'utilizzo di un unico test che rivela e genotipizza il virus;

per lo screening secondario è opportuno usare una metodica in grado di rilevare e genotipizzare l'HPV in quanto l'alta prevalenza dell'HPV in questo screening, e le alterazioni cito-istologiche correlate, giustificano l'impiego di un unico test in grado di rilevare l'infezione e genotipizzarla. Il costo maggiore del test è ammortizzato dal minor impegno di risorse umane e strumentali necessari per ripetere il test sui positivi (che sono più del $50 \%$ dei casi totali).

Infine, il test di cellularità per la valutazione dell'idoneità del preparato anche per la ricerca del virus HPV (tabella 9), ha mostrato una differenza di positività (cut-off di 300000 cellule/prelievo) statisticamente significativa tra i campioni idonei e non idonei.

Dati preliminari ottenuti dalla comparazione della

Tabella I. Studio pilota MSTI: primers utilizzati per la PCR multiplex home-made

\begin{tabular}{|c|c|c|c|c|c|}
\hline MICRORGANISMO & $\begin{array}{l}\text { SEQUENZA FORWARD } \\
\text { SEQUENZA REVERSE }\end{array}$ & BIBLIOGRAFIA & $\begin{array}{l}\text { LUNGHEZZA } \\
\text { FRAMMENTO }\end{array}$ & TARGET & $\mathrm{T}$ annealing \\
\hline $\mathrm{CT}$ & $\begin{array}{l}\text { 5'CTAGGCGTTTGTACTCCGTCA3' } \\
\text { 5'TCCTCAGGAGTTTATGCACT3' }\end{array}$ & $(25)$ & $200 \mathrm{bp}$ & $\begin{array}{l}\text { ORF8 } \\
\text { plasmide criptico }\end{array}$ & $54^{\circ} \mathrm{C}$ \\
\hline NG & $\begin{array}{l}\text { 5’ACTGCGTTCTGAACTGGGTG3' } \\
\text { 5'GGCGGTCAATTTCACGCG3' }\end{array}$ & $(25)$ & $281 \mathrm{bp}$ & rRNA I6S & $54^{\circ} \mathrm{C}$ \\
\hline Ureaplasma spp & $\begin{array}{l}\text { 5'GAAACGACGTCCATAAGCAACT3' } \\
\text { 5'GCAATCTGCTCGTGAAGTATTAC3 }\end{array}$ & $(25)$ & $423 \mathrm{bp}$ & Ureasi - subunità â & $54^{\circ} \mathrm{C}$ \\
\hline $\mathrm{MH}$ & $\begin{array}{l}\text { 5'CAATGGCTAATGCCGGATACGC3' } \\
\text { 5'GGTACCGTCAGTCTGCAA3' }\end{array}$ & $(15)$ & $334 \mathrm{bp}$ & rRNA I6S & $56^{\circ} \mathrm{C}$ \\
\hline $\begin{array}{l}\text { CONTROLLO } \\
\text { INTERNO }\end{array}$ & $\begin{array}{l}\text { 5'CCACGAAACTACCTTCAAC3' } \\
\text { 5'GATCTTCATTGTGCTGGG3' }\end{array}$ & $(3 I)$ & $158 \mathrm{bp}$ & B-Actina & $56-54^{\circ} \mathrm{C}$ \\
\hline
\end{tabular}


cellularità valutata su vetrino (Pap-test) e calcolata con la metodica RT-PCR indicano che la cellularità di un tampone cervicale dovrebbe essere aumentata ad almeno 500000 cellule/prelievo anche in relazione ad un incremento della cellularità media osservata $(4256732 \pm 456678$ cellule/prelievo) con l'inserimento nel referto della cellularità e relativa idoneità del prelievo. L'inserimento di un cut-off di cellularità, parametro che comunque non viene valutato dai test di screening attualmente più utilizzati, come il test $\mathrm{HC} 2$, aumenta il numero di positivi rilevati ma non permette di valutare la presenza di cellule endocervicali (cellule bersaglio del virus) discriminandole da altri tipi di cellule (come quelle di tipo infiammatorio). Questo rimane un limite della metodica molecolare utilizzata a sé stante anche se l'indice di cellularità si sta rilevando un buon parametro per la valutazione della correttezza del prelievo.

Tabella 2. A: Numero di casi positivi riscontrati nei campioni dello studio pilota MSTI relativo a ciascun patogeno; B: Frequenza relativa

\begin{tabular}{llllll}
\hline A & COLTURALE & IST2 & Probe Tec BD & multiplex PCR & RT-PCR \\
\hline C. trachomatis & - & - & 12 & 13 & I7 \\
\hline Ureaplasma spP & 39 & 72 & - & 88 & I25 \\
\hline M. hominis & 8 & 15 & - & 22 & 34 \\
\hline N. gonorrhoeae & 4 & - & - & 22 & 12 \\
\hline B & COLTURALE & IST2 & Probe Tec BD & multiplex PCR & RT-PCR \\
\hline C. trachomatis & - & - & 4.00 & 4.33 & 5.67 \\
\hline Ureaplasma sPP & 13.00 & 24.00 & - & 29.33 & $4 I .67$ \\
\hline M. hominis & 2.67 & 5.00 & - & 7.33 & 11.33 \\
\hline N. gonorrhoeae & 1.33 & - & - & 7.33 & 4.00 \\
\hline
\end{tabular}

Tabella 3. Studio pilota MSTI: confronto delle sensibilità diagnostiche relative a ciascuna metodica utilizzata

\begin{tabular}{llllll}
\hline & COLTURALE & IST2 & Probe Tec BD & multiplex PCR & RT-PCR \\
\hline C. trachomatis & - & - & 0.88 & 0.93 & I.00 \\
\hline Ureaplasma sPP & 0.31 & 0.58 & - & 0.70 & 1.00 \\
\hline M. hominis & 0.24 & 0.44 & - & 0.65 & 1.00 \\
\hline N. gonorrhoeae & 0.33 & - & - & 0.92 & 1.00 \\
\hline
\end{tabular}

Tabella 4. Studio MST2: frequenza delle principali MST rilevate nei diversi tipi di campione (A: maschi, B: femmine)

\begin{tabular}{|c|c|c|c|c|c|c|c|c|}
\hline \multirow[t]{2}{*}{ A } & \multicolumn{2}{|c|}{$\begin{array}{l}\text { LIQUIDO SEMINALE } \\
\text { ( } 110 \text { campioni) }\end{array}$} & \multicolumn{2}{|c|}{$\begin{array}{l}\text { TAMP URETRALE } \\
\text { (106 campioni) }\end{array}$} & \multicolumn{2}{|c|}{$\begin{array}{c}\text { URINE } \\
\text { (90 campioni) }\end{array}$} & \multicolumn{2}{|c|}{$\begin{array}{c}\text { TOTALI } \\
\text { (306 campioni) }\end{array}$} \\
\hline & casi positivi & $\%$ & casi positivi & $\%$ & casi positivi & $\%$ & casi positivi & $\%$ \\
\hline$\overline{\mathrm{CT}}$ & 18 & 16.36 & 25 & 23.58 & 5 & 5.56 & 48 & 15.68 \\
\hline UU & 6 & 5.45 & 6 & 5.66 & 7 & 7.78 & 19 & 6.21 \\
\hline UP & 20 & 18.18 & 13 & 12.26 & $\mathrm{II}$ & 12.22 & 44 & 14.38 \\
\hline$\overline{\mathrm{MH}}$ & 9 & 8.18 & 4 & 3.77 & 4 & 4.44 & 17 & 5.56 \\
\hline$M G$ & 3 & 2.73 & 7 & 6.60 & 0 & 0.00 & 10 & 3.27 \\
\hline \multirow[t]{2}{*}{$\bar{B}$} & \multicolumn{2}{|c|}{$\begin{array}{l}\text { MAT. CERVICALE } \\
\text { (553 campioni) }\end{array}$} & \multicolumn{2}{|c|}{$\begin{array}{l}\text { TAMP URETRALE } \\
\text { (29 campioni) }\end{array}$} & \multicolumn{2}{|c|}{$\begin{array}{c}\text { URINE } \\
\text { (45 campioni) }\end{array}$} & \multicolumn{2}{|c|}{$\begin{array}{c}\text { TOTALI } \\
\text { (627 campioni) }\end{array}$} \\
\hline & casi positivi & $\%$ & casi positivi & $\%$ & casi positivi & $\%$ & casi positivi & $\%$ \\
\hline$\overline{\mathrm{CT}}$ & 30 & 5.42 & $\mathrm{I}$ & 3.45 & 1 & 2.22 & 32 & 5.1 \\
\hline UU & 62 & 11.21 & 4 & 13.79 & 0 & 0.00 & 66 & 10.53 \\
\hline UP & 194 & 35.08 & 8 & 27.59 & 13 & 28.89 & 215 & 34.29 \\
\hline $\mathrm{MH}$ & 61 & 11.03 & 1 & 3.45 & 3 & 6.67 & 65 & 10.37 \\
\hline$M G$ & 10 & 1.81 & I & 3.45 & 0 & 0.00 & I I & 1.75 \\
\hline
\end{tabular}

Tabella 5. Studio MST2: coinfezioni statisticamente significative con relativo valore del $\chi^{2}$

\begin{tabular}{lccc}
\hline Coinfezione & Casi con presenza di coinfezione & $\chi^{2}$ & $p v a l u e$ \\
\hline CT-MG & 75 & 6.36 & $p<0.025$ \\
\hline UU-UP & 37 & 11.60 & $p<0.005$ \\
\hline UU-MH & 16 & 11.75 & $p<0.005$ \\
\hline UP-MH & 34 & 8.42 & $p<0.005$ \\
\hline
\end{tabular}


Tabella 6. Studio HPV e MSTI: Casi positivi ad HPV rilevati nelle due popolazioni in studio, con relativa analisi citologica. Screening primario 300 campioni

\begin{tabular}{|c|c|c|c|c|c|c|c|c|c|c|c|}
\hline & \multicolumn{2}{|c|}{ Nessuna alterazione, n (\%) } & \multicolumn{2}{|c|}{ ASCUS, n (\%) } & \multicolumn{2}{|c|}{ L-SIL, n (\%) } & \multicolumn{2}{|c|}{ H-SIL, n (\%) } & \multirow{2}{*}{$\begin{array}{c}\text { TOTALE } \\
248\end{array}$} & $x^{2}$ & \multirow[t]{2}{*}{ pvalue } \\
\hline HPV - & 234 & $(94.35)$ & $\mathrm{II}$ & $(4.43)$ & 3 & $(\mathrm{I} .2 \mathrm{I})$ & - & - & & & \\
\hline $\mathrm{HPV}+$ & 39 & (75) & 2 & $(3.85)$ & 7 & (13.46) & 4 & (7.69) & 52 & & \\
\hline Totale & 273 & & 13 & & 10 & & 4 & & 300 & & \\
\hline \multicolumn{12}{|c|}{ Screening secondario || 40 campioni } \\
\hline & \multicolumn{2}{|c|}{ Nessuna alterazione, n (\%) } & \multicolumn{2}{|c|}{ ASCUS, n (\%) } & \multicolumn{2}{|c|}{ L-SIL, n (\%) } & \multicolumn{2}{|c|}{ H-SIL, n (\%) } & TOTALE & & \\
\hline HPV - & - & - & 441 & $(79.17)$ & 112 & $(20.11)$ & 4 & $(0.72)$ & 557 & & \\
\hline$\underline{\mathrm{HPV}}+$ & - & - & 132 & $(22.64)$ & 394 & $(67.58)$ & 57 & $(9.78)$ & 583 & & \\
\hline Totale & 0 & & 573 & & 506 & & 61 & & 1140 & II & $<0.005$ \\
\hline
\end{tabular}

Tabella 7. Studio MST2: correlazioni significative riscontrate tra infezione ed età, con relativi valori del $\chi^{2}$ e probabilità associata - soggetti di sesso femminile.

\begin{tabular}{|c|c|c|c|c|c|}
\hline \multirow{2}{*}{\multicolumn{2}{|c|}{ C. trachomatis }} & \multirow{2}{*}{$\begin{array}{l}\text { Casi totali } \\
627\end{array}$} & \multirow{2}{*}{$\begin{array}{l}\text { Casi positivi } \\
32\end{array}$} & \multirow{2}{*}{$\begin{array}{l}\text { Frequenza (\%) } \\
5.1\end{array}$} & \multirow[b]{3}{*}{ pvalue } \\
\hline & & & & & \\
\hline & Totale campioni & Casi positivi osservati & Frequenza (\%) & $x^{2}$ & \\
\hline$<23$ & 51 & 3 & 5.88 & & \\
\hline $23-32$ & 155 & 17 & 10.97 & & \\
\hline $33-42$ & 260 & 8 & 3.08 & & \\
\hline $43-52$ & 96 & 3 & 3.125 & & \\
\hline$>52$ & 65 & $\mathrm{I}$ & 1.54 & 15.76 & $<0.005$ \\
\hline \multirow{2}{*}{\multicolumn{2}{|c|}{ U. urealyticum }} & Casi totali & Casi positivi & Frequenza (\%) & \\
\hline & & 627 & 66 & 10.53 & \\
\hline & Totale campioni & Casi positivi osservati & Frequenza (\%) & $x^{2}$ & pvalue \\
\hline$<23$ & 51 & 11 & 21.57 & & \\
\hline $23-32$ & 155 & 19 & 12.26 & & \\
\hline $33-42$ & 260 & 19 & 7.31 & & \\
\hline $43-52$ & 96 & 11 & 11.46 & & \\
\hline$>52$ & 65 & 6 & 9.23 & 10.16 & $<0.05$ \\
\hline
\end{tabular}

Tabella 8. Studio HPV e MSTI: analisi statistica della distribuzione della frequenza dell'infezione da HPV in relazione all'età. Screening primario Screening secondario

\begin{tabular}{|c|c|c|c|c|c|c|c|}
\hline $\begin{array}{c}\text { Totale } \\
\text { campioni }\end{array}$ & $\begin{array}{c}\text { Casi } \\
\text { positivi }\end{array}$ & $\chi^{2}$ & pvalue & $\begin{array}{c}\text { Totale } \\
\text { campioni }\end{array}$ & $\begin{array}{c}\text { Casi } \\
\text { positivi }\end{array}$ & $\chi^{2}$ & pvalue \\
\hline
\end{tabular}

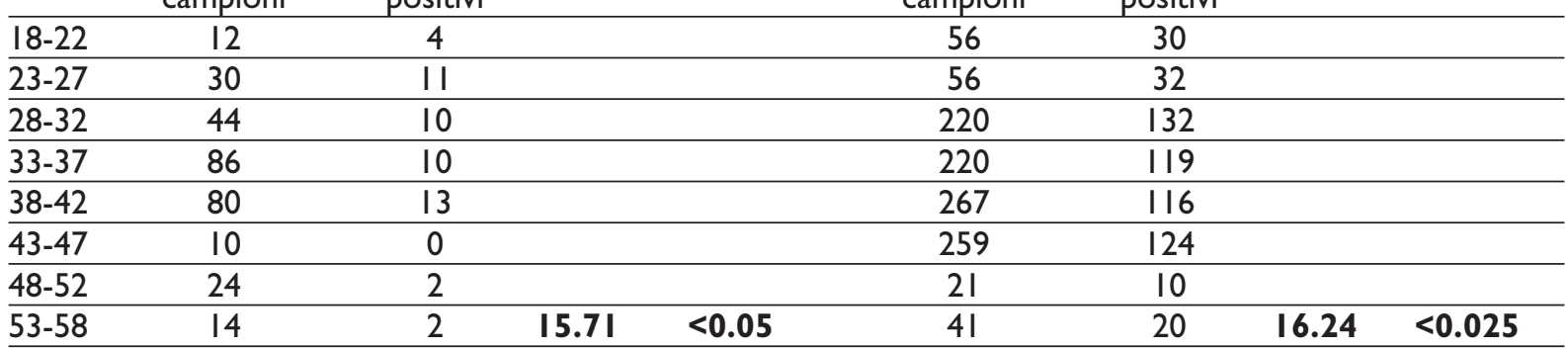

Tabella 9. Studio MST2 e HPV: valori di cellularità media, deviazione standard, errore standard e intervallo di confidenza al $95 \%$ ottenuti per ogni tipo di campione.

\begin{tabular}{|c|c|c|c|c|c|c|}
\hline Tipo di campione & $\mathrm{C}_{\mathrm{m}}$ & $\begin{array}{l}\text { deviazione } \\
\text { standard }\end{array}$ & $\begin{array}{l}\text { errore } \\
\text { standard }\end{array}$ & $\begin{array}{c}\text { intervallo di confidenza } \\
(95 \%)\end{array}$ & CUT-OFF & $\%<$ CUT-OFF \\
\hline $\begin{array}{l}\text { ThinPrep } \\
\text { (cell/prelievo) }\end{array}$ & 1015824 & 1373476 & 87392 & $1015824 \pm 174784$ & 300000 & 19.33 \\
\hline $\begin{array}{l}\text { Tampone cervicale } \\
\text { (cell/prelievo) }\end{array}$ & $23747 I I$ & 3667557 & 155960 & $23747 \mid I \pm 311921$ & 300000 & 13.47 \\
\hline $\begin{array}{l}\text { Tampone uretrale } \\
\text { (cell/prelievo) }\end{array}$ & 1684616 & 2263557 & 194816 & $1684616 \pm 389632$ & 150000 & $|4.8|$ \\
\hline Urine (cell/ml) & 396495 & 701471 & 60373 & $396495 \pm 120746$ & 40000 & 26.67 \\
\hline
\end{tabular}




\section{BIBLIOGRAFIA}

1. Abele-Horn M, Kopp A, Sternberg U, et al. Polymerase chain reaction versus culture for detection of Ureaplasma urealyticum and Mycoplasma hominis in the urogenital tract of adults and the respiratory tract of newborns. Eur J Clin Microbiol Infect Dis 1996; 15: 595-8.

2. Amirmozafari N, Mirnejad R, Kazemi B, Sariri E, Bojari MR, Darkahi FD.Comparison of polymerase chain reaction and culture for detection of genital mycoplasma in clinical samples from patients with genital infections. Saudi Med J 2009; 11: 1401-5.

3. Arbyn M, Anttila A, Jordan J, et al. European Guidelines for Quality Assurance in Cervical Cancer Screening. Second Edition-Summary Document. Ann Oncol 2010; 21: 448-58.

4. Babady NE, Mead P, Stiles J, et al. Comparison of the Luminex xTAG RVP Fast assay and the Idaho Technology FilmArray RP assay for detection of respiratory viruses in pediatric patients at a cancer hospital. J Clin Microbiol 2012; 7: 2282-8.

5. Baczynska A, Hvid M, Lamy P, Birkelund S, Christiansen G, Fedder J. Prevalence of Mycoplasma genitalium, Mycoplasma hominis and Chlamydia trachomatis among Danish patients requesting abortion. Syst Biol Reprod Med 2008; 3: 127-34.

6. Bébéar C, de Barbeyrac B. Genital Chlamydia trachomatis infections. Clin Microbiol Infect 2009; 1: 4-10.

7. Beebe JL, Gershman KA, Kelley JK, Hagner D, Creede P. How adequate is adequate for the collection of endocervical specimens for Chlamydia trachomatis testing? Sex Transm Dis 1999; 10: 579-83.

8. Bignell C; IUSTI/WHO. 2009 European (IUSTI/WHO) Guideline on the Diagnosis and Treatment of Gonorrhoea in Adults. Int J STD AIDS 2009; 20: 453-7.

9. Bulkmans NW, Rozendaal L, Snijders PJ, et al. POBASCAM, a population-based randomised controlled trial for implementation of high-risk HPV testing in cervical screening. Int J Cancer 2004; 110: 94-110.

10. Cattani P, Zannoni GF, Ricci C, Cattani P, et al. Clinical performance of human papillomavirus E6 and E7 mRNA testing for high-grade lesions of the cervix. J Clin Microbiol 2009; 12: 3895-901.

11. Cook RL, Hutchison SL, Østergaard L, Braithwaite RS, Ness RB. Systematic review: noninvasive testing for Chlamydia trachomatis and Neisseria gonorrhoeae. Ann Intern Med 2005; 142: 914-25.

12. Currie MJ, McNiven M, Yee T, Schiemer U, Bowden FJ. Pooling of clinical specimens prior to testing for Chlamydia trachomatis by PCR is accurate and cost saving. J Clin Microbiol 2004; 10: 4866-7.

13. Cuzick J, Clavel C, Petry KU, et al. Overview of the European and North American studies on HPV testing in primary cervical cancer screening. Int $J$ Cancer 2006; 119: 1095-101.

14. Cuzick J, Cadman L, Mesher D, et al. Comparing the performance of six human papillomavirus tests in a screening population. Br J Cancer 2013; 4: 908-13.

15. Egawa T, Morioka I, Morisawa T, et al. Ureaplasma urealyticum and Mycoplasma hominis presence in umbilical cord is associated with pathogenesis of funisitis. Kobe J Med Sci 2007; 5: 241-9.

16. Falk L, Coble BI, Mjörnberg PA, Fredlund $\mathrm{H}$. Sampling for Chlamydia trachomatis infection - a comparison of vaginal, first-catch urine, combined vaginal and first-catch urine and endocervical sampling. Internat J STD \& AIDS 2010; 21: 56-62.

17. Gaydos CA, Ferrero DV, Papp J. Laboratory aspects of screening men for Chlamydia trachomatis in the new millennium. Sex Transm Dis 2008; 35: S45-S50.

18. Gdoura R, Kchaou W, Chaari C, et al. Ureaplasma urealyticum, Ureaplasma parvum, Mycoplasma hominis and Mycoplasma genitalium infections and semen quality of infertile men. BMC Infect Dis 2007; 7: 129-37.

19. Gravitt PE, Schiffman M, Solomon D, Wheeler CM, Castle PE. A comparison of linear array and hybrid capture 2 for detection of carcinogenic human papillomavirus and cervical precancer in ASCUSLSIL triage study. Cancer Epidemiol Biomarkers Prev 2008; 5: 1248-54

20. Gupta A, Gupta A, Gupta S, Mittal A, Chandra P, Gill AK. Correlation of mycoplasma with unexplained infertility. Arch Gynecol Obstet Mar 2009; 6: 981-5.

21. Imudia AN, Detti L, Puscheck EE, Yelian FD, Diamond MP. The prevalence of Ureaplasma urealyticum, Mycoplasma hominis, Chlamydia trachomatis and Neisseria gonorrhoeae infections, and the rubella status of patients undergoing an initial infertility evaluation. $J$ Assist Reprod Genet 2008; 1: 43-6.

22. Joste NE, Kundsin RB, Genest DR. Histology and Ureaplasma urealyticum culture in 63 cases of first trimester abortion. Am J Clin Pathol 1994; 6: 729-32.

23. Lanjouw E, Ossewaarde JM, Stary A, Boag F, van der Meijden WI. 2010 European Guideline for the management of Chlamydia trachomatis infections. Int J STD AIDS 2010; 11: 729-37.

24. Larsen B, Hwang J. Mycoplasma, Ureaplasma and adverse pregnancy outcomes: a fresh look. Infect Dis Obstet Gynecol 2010: 521921-7.

25. Lee SR, Chung JM, Kim YG. Rapid one step detection of pathogenic bacteria in urine with sexually transmitted disease (STD) and prostatitis patient by multiplex PCR assay (mPCR). J Microbiol 2007; 45 (5): 453-9.

26. Leveque N, Van Haecke A, Renois F, Boutolleau D, Talmud D, Andreoletti L. Rapid virological diagnosis of central nervous system infections by use of a multiplex reverse transcription-PCR DNA microarray. J Clin Microbiol 2011; 11: 3874-9.

27. Loeffelholz MJ, Jirsa SJ, Teske RK, Woods JN. Effect of endocervical specimen adequacy on ligase chain reaction detection of Chlamydia trachomatis. J Clin Microbiol 2001; 11: 3838-41.

28. Luki N, Lebel P, Boucher M, Doray B, Turgeon J, Brousseau R. Comparison of polymerase chain reaction assay with culture for detection of genital mycoplasmas in perinatal infections. Eur $J$ Clin Microbiol Infect Dis 1998; 4: 255-63.

29. Mancini N, Carletti S, Ghidoli N, Cichero P, Burioni $\mathrm{R}$, Clementi M. The era of molecular and other nonculture-based methods in diagnosis of sepsis. Clin Microbiol Rev 2010; 1: 235-51.

30. Mayrand MH, Duarte-Franco E, Rodrigues I, et al. Human papillomavirus DNA versus Papanicolau screening tests of cervical cancer. $N$ England $J$ Med 2007; 16: 1578-88.

31. Mochizuki H, Nomura T, Kawamura I, Mitsuyama M. Enhanced resistance to Gram-positive bacterium and increased susceptibility to bacterial endotoxin in mice sensitized with Propionibacterium acnes: involvement of Toll-like receptor. FEMS Immunol Med Microbiol 2005; 2: 287-93.

32. Morré SA, Ouburg S, van Agtmael MA, de Vries HJ. Lymphogranuloma venereum diagnostics: from culture to real-time quadriplex polymerase chain 
reaction. Sex Transm Infect 2008; 84: 252-3.

33. Morris BJ. Cervical human papillomavirus screening by PCR: advantages of targeting the E6/E7 region. Clin Chem Lab Med 2005; 11: 1171-7.

34. Ouzounova-Raykova VV, Markovska R, Mizgova G, Mitov IG. Detection of the sexually transmissible genital mycoplasmas by polymerase chain reaction in women. Sex Health 2011; 3: 445-6.

35. Poljak M, Kocjan BJ. Commercially available assays for multiplex detection of alpha human papillomaviruses. Expert Rev Anti Infect Ther 2010; 10: 1139-62.

36. Rodrigues MM, Fernandes PÁ, Haddad JP, et al. Frequency of Chlamydia trachomatis, Neisseria gonorrhoeae, Mycoplasma genitalium, Mycoplasma hominis and Ureaplasma species in cervical samples. J Obstet Gynaecol 2011; 3: 237-41.

37. Ronco G, Segnan N, Giorgi-Rossi P, et al. Human papillomavirus testing and liquid-based cytology in primary cervical screening: results at recruitment from the NTCC randomized controlled trial. J Natl Cancer Inst 2006; 98: 765-74.

38. Ross JDC, Jensen JS. Mycoplasma genitalium as a sexually transmitted infection: implication for screening, testing, and treatment. Sex Transm Infect 2006; 82: 269-71.

39. Salari MH, Karimi A. Prevalence of Ureaplasma urealyticum and Mycoplasma genitalium in men with non-gonococcal urethritis. East Mediterr Health $J$ 2003; 3: 291-5.

40. Shipitsyna E, Shalepo K, Savicheva A, Unemo M, Domeika M. Pooling samples: the key to sensitive, specific and cost-effective genetic diagnosis of Chlamydia trachomatis in low- resource countries. Acta Derm Venereol 2007; 87: 140-3.

41. Stellrecht KA, Woron AM, Mishrik NG, Venezia RA. Comparison of multiplex PCR assay with culture for detection of genital mycoplasmas. J Clin Microbiol 2004; 4: 1528-33.
42. Szarewski A, Mesher D, Cadman L, et al. Comparison of Seven Tests for High-Grade Cervical Intraepithelial Neoplasia in Women with Abnormal Smears: the Predictors 2 Study. J Clin Microbiol 2012; 6: 1867-73.

43. Takahashi S, Takeyama K, Miyamoto S, et al. Detection of Mycoplasma genitalium, Mycoplasma hominis, Ureaplasma urealyticum, and Ureaplasma parvum DNAs in urine from asymptomatic healthy young Japanese men. J Infect Chemother 2006; 5: 269-71.

44. Teng K, Li M, Yu W, Li H, Shen D, Liu D. Comparison of PCR with culture for detection of Ureaplasma urealyticum in clinical samples from patients with urogenital infections. J Clin Microbiol 1994; 9: 2232-4.

45. Van Dyck E, Ieven M, Pattyn S, Van Damme L, Laga M. Detection of Chlamydia trachomatis and Neisseria gonorrhoeae by enzyme immunoassay, culture and three nucleic acid amplification tests. J Clin Microbiol 2001; 39: 1751-6.

46. Viscardi RM. Ureaplasma species: role in diseases of prematurity. Clin Perinatol 2010; 2: 393-409.

47. Welsh LE, Quinn TC, Gaydos CA. Influence of endocervical specimen adequacy on PCR and direct fluorescent-antibody staining for detection of Chlamydia trachomatis infections. J Clin Microbiol 1997; 12: 3078-81.

48. WHO. Global strategy for prevention and control of sexually transmitted infection: 2006-2015. World Health Organization, 2007, Ginevra, Svizzera.

49. Witt A, Berger A, Gruber CJ, et al. Increased intrauterine frequency of Ureaplasma urealyticum in women with preterm labor and preterm premature rupture of the membranes and subsequent cesarean delivery. Am J Obstet Gynecol 2005; 5: 1663-9.

50. Zeighami H, Peerayeh SN, Yazdi RS, Sorouri R. Prevalence of Ureaplasma urealyticum and Ureaplasma parvum in semen of infertile and healthy men. Int J STD AIDS 2009; 6: 387-90. 